\title{
ON THE ORBITAL INCLINATION OF PROXIMA CENTAURI b
}

\author{
Stephen R. Kane ${ }^{1}$, Dawn M. Gelino ${ }^{2}$, and Margaret C. Turnbull ${ }^{3}$ \\ ${ }^{1}$ Department of Physics \& Astronomy, San Francisco State University, 1600 Holloway Avenue, San Francisco, CA 94132, USA; skane@sfsu.edu \\ ${ }^{2}$ NASA Exoplanet Science Institute, Caltech, MS 100-22, 770 South Wilson Avenue, Pasadena, CA 91125, USA \\ ${ }^{3}$ SETI Institute, Carl Sagan Center for the Study of Life in the Universe, Off-Site: 2801 Shefford Drive, Madison, WI 53719, USA \\ Received 2016 October 26; revised 2016 December 1; accepted 2016 December 5; published 2017 January 9
}

\begin{abstract}
The field of exoplanetary science has seen discovery rates increase dramatically over recent years, due largely to the data from the Kepler mission. Even so, individual discoveries of planets orbiting nearby stars are very important for studies of characterization and near-term follow-up prospects. The recent discovery of a terrestrial planet candidate orbiting Proxima Centauri presents numerous opportunities for studying a super-Earth within our own stellar backyard. One of the remaining ambiguities of the discovery is the true mass of the planet since the discovery signature was obtained via radial velocities. Here, we describe the effect of orbital inclination on the Proxima Centauri planet, in terms of mass, radius, atmosphere, and albedo. We calculate the astrometric, angular separation, and reflected light properties of the planet including the effects of orbital eccentricity. We further provide dynamical simulations that show how the presence of additional terrestrial planets within the Habitable Zone varies as a function of inclination. Finally, we discuss these effects in the context of future space-based photometry and imaging missions that could potentially detect the planetary signature and resolve the inclination and mass ambiguity of the planet.
\end{abstract}

Key words: astrobiology - planetary systems - stars: individual (Proxima Centauri) techniques: high angular resolution

\section{INTRODUCTION}

Exoplanet discoveries have increasingly focused on terrestrial planets as detection capabilities continue to improve. For example, the planet yield from the Kepler mission that is of primary interest is the yield for terrestrial planets that lie in the Habitable Zone (HZ) of their host stars (Kane et al. 2016). For non-transiting planets, the radial velocity (RV) method continues to be the primary method to detect terrestrial planets suitable for follow-up characterization. For example, the star HD 40307 harbors a system of super-Earths discovered using the RV technique (Mayor et al. 2009), one of which is known to lie within the HZ of the star (Tuomi et al. 2013).

The closest exoplanet to the solar system was recently identified by Anglada-Escudé et al. (2016). This exoplanet is orbiting the closest star, Proxima Centauri. Proxima is a latetype flare star with a rotation period of $\sim 84$ days confirmed photometrically (Anglada-Escudé et al. 2016) and spectroscopically (Collins et al. 2016; Robertson et al. 2016). The associated planet was detected through a long-term RV campaign and found to have an orbital period of 11.186 days, a semimajor axis of $0.0485 \mathrm{au}$, and a minimum mass $\sim 30 \%$ larger than the Earth. Formation scenarios for the planet include possible perturbations from close encounters with the Alpha Centauri stellar components as a possible explanation for the relatively high planetary orbital eccentricity (Barnes et al. 2016; Coleman et al. 2016). The size of the planet remains unknown since transits have been effectively ruled out (Anglada-Escudé et al. 2016; Davenport et al. 2016; Kipping et al. 2016). However, even though the inclination, true mass, and radius are unknown, the planet is likely terrestrial. This has led to the exploration of potential habitability conditions and detectable biosignatures (Barnes et al. 2016; Meadows et al. 2016; Ribas et al. 2016; Turbet et al. 2016), including the prospect of life in high-UV environments (O'Malley-James \& Kaltenegger 2016).
Here we present an investigation of the effects of the inclination of the Proxima Centauri b orbital plane relative to the line of sight. The effects of the inclination on the mass of the planet and related physical properties are described in Section 2. The astrometric signature of the planet as a function of orbital inclination is considered in Section 3. In Section 4, we provide calculations of the star-planet angular separation as a function of inclination and orbital phase. Section 5 discusses the dependence of inclination on the expected phase variations due to reflected light and related effects. Section 6 presents the results of a dynamical simulation that constrains the presence of other potential terrestrial planets within the HZ of the host star. In Section 7 we discuss observable imaging signatures of the planet and mission requirements to achieve a detection.

\section{THE EFFECT OF INCLINATION ON PLANETARY PROPERTIES}

The minimum mass of the Proxima planet measured from the RV work of Anglada-Escudé et al. (2016) is $M_{p} \sin i=1.27$ Earth masses. The range of masses and radii for which a planet can reasonably be expected to be terrestrial has been studied in detail, thanks largely to the planet yield from the Kepler mission (Weiss \& Marcy 2014; Dressing et al. 2015; Rogers 2015). Many of these studies find that there is evidence of a density transition that occurs at $\sim 1.5-2.0$ Earth radii $\left(R_{\oplus}\right)$, whereby the composition of objects larger than this becomes dominated by volatile rather than refractory materials. Using the mass-radius relationship of Weiss \& Marcy (2014), we estimate that this transition corresponds to 3.9-5.1 Earth masses $\left(M_{\oplus}\right)$. In order for the mass to exceed this range, the orbital inclination would need to satify $i<14.4$. Assuming randomly oriented orbits, and excluding the $1.5 \%$ transit probability (Anglada-Escudé et al. 2016), the probability that the planet lies in the terrestrial regime is $\sim 84 \%$. 


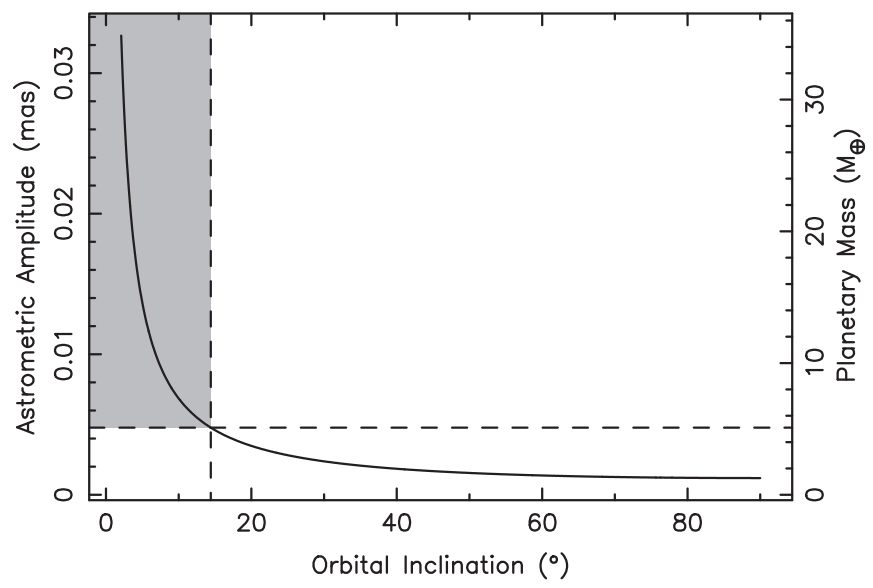

Figure 1. Astrometric amplitude of Proxima Centauri due to the orbit of the planet as a function of orbital inclination. The corresponding planetary mass is shown on the right-hand $y$-axis of the plot. The dashed cross-hairs indicate the location where the mass is at the terrestrial/gas planet threshold (see Section 2). The gray shaded region is then where an astrometric detection would confirm that the planet is too massive to be considered terrestrial.

Apart from increasing the radius of the planet, decreasing the orbital inclination, and thus increasing the planetary mass, also has an effect on the atmospheric properties (see Madhusudhan et al. 2016 and references therein). For a given insolation flux, the atmospheric composition will determine the resulting chemistry and the formation of reflective layers in the upper atmospheric layers. Of particular relevance is the transition from terrestrial to giant planet whereby the dominant atmospheric components change from heavy molecules $\left(\mathrm{H}_{2} \mathrm{O}, \mathrm{CO}_{2}\right.$, $\mathrm{N}_{2}$ ) to high $\mathrm{H} / \mathrm{He}$ abundances. The impact of these on observations lies primarily in the affect on the resulting albedo and contrast ratios of the planet to the host star. These factors are discussed in more detail in Sections 5 and 7.

\section{ASTROMETRIC SIGNATURE}

A change in planetary mass has implications for the expected astrometric signature. The amplitude of an astrometric signature is given by

$$
\alpha=\left(\frac{M_{p}}{M_{\star}}\right)\left(\frac{a}{1 \mathrm{au}}\right)\left(\frac{d}{1 \mathrm{pc}}\right)^{-1} \operatorname{arcsec},
$$

where $M_{p}$ and $M_{\star}$ are the planetary and stellar masses respectively, $a$ is the semimajor axis in astronomical units, and $d$ is the distance to the system. In Figure 1 we plot the astrometric signature of the Proxima planet as a function of the orbital inclination. We include the corresponding mass of the planet on the right-hand $y$-axis of the plot and the location of the terrestrial/gas planet threshold (dashed lines), as discussed in Section 2. The gray region of the plot thus highlights the region where an astrometric detection of that magnitude would resolve the $\sin i$ ambiguity in favor of the planet being nonterrestrial in nature.

The Gaia mission (Prusti et al. 2016) is currently in the process of astrometric data releases (Brown et al. 2016; Lindegren et al. 2016). ${ }^{4}$ These data will undoubtedly contribute enormously to exoplanet science. The exoplanet detection capabilities of Gaia have been previously investigated by

\footnotetext{
http://gea.esac.esa.int/archive
}

Perryman et al. (2014). The expected science performance of Gaia has been described by de Bruijne (2012) and is also available at the European Space Agency web site for the mission. ${ }^{5}$ From these sources, the expected astrometric precision for bright $(5<V<14) \mathrm{M}$ dwarf stars is 5-16 $\mu$ as. This is more than sufficient to adequately sample the gray region of Figure 1 and perhaps detect the planetary signature within the terrestrial regime. A limitation of such analysis is the relatively short orbital period of the planet in comparison to the cadence of the Gaia observations. However, combining the astrometry with further RVs will resolve the full orbital solution for the planet (Tuomi et al. 2009).

\section{ANGULAR STAR-PLANET SEPARATION}

The Proxima Centauri system is likely to be an attractive target for planned imaging missions and the angular separation of the planet from the host star will be a key part of those observations. The planning of those observations is particularly important if the eccentricity of the planetary orbit is indeed close to the upper limit of $e=0.35$ found by Anglada-Escudé et al. (2016), since even face-on orbits will have a time dependence on the star-planet separation. It is also worth noting that there is a bias toward higher orbital eccentricities in RV exoplanet surveys (Zakamska et al. 2011), thus increasing the likelihood of an eccentricity for Proxima Centauri $b$ that lies closer to the maximum value. Using the methodology of Kane (2013), we calculate the angular separation of the planet over one complete orbit. Shown in Figure 2 are the projected and angular separations of the planet from the host star for four possible orbital inclinations, including edge-on $\left(i=90^{\circ}\right)$ and face-on $\left(i=0^{\circ}\right)$ viewing angles. An orbital phase of zero corresponds to the location of superior conjunction. These calculations assume both the eccentricity of $e=0.35$ and the argument of periastron of $\omega=310^{\circ}$ given by Anglada-Escudé et al. (2016).

As expected, the maximum angular star-planet separation ( $\sim 65$ mas) occurs for the case of $i=0^{\circ}$. However, this separation is only slightly larger than those of other inclinations. The primary consideration for the different orbital inclinations is the timing of the observations, which can result in negligible star-planet separations, particularly for $i>60^{\circ}$. This is discussed in the context of future missions in Section 7.

\section{REFLECTED LIGHT AND PHASE VARIATIONS}

Since observations have not currently shown that the Proxima Centauri planet transits the host star (Anglada-Escudé et al. 2016; Davenport et al. 2016; Kipping et al. 2016), detailed characterization of the atmosphere will likely rely largely upon reflected/scattered light. The dependence of photometric phase variations due to reflected light on planetary radii and albedo is well known (Seager et al. 2000; Sudarsky et al. 2005) and has also been shown to depend on orbital eccentricity (Kane \& Gelino 2010). Kane \& Gelino (2011a) further demonstrated how phase variations depend on orbital inclination, providing a possible mechanism to distinguish between different classes of orbital objects (Kane \& Gelino 2012a). Lovis et al. (2016) have calculated phase amplitudes and contrast ratios for direct detection of the planet

\footnotetext{
5 http://www.cosmos.esa.int/web/gaia/science-performance
} 

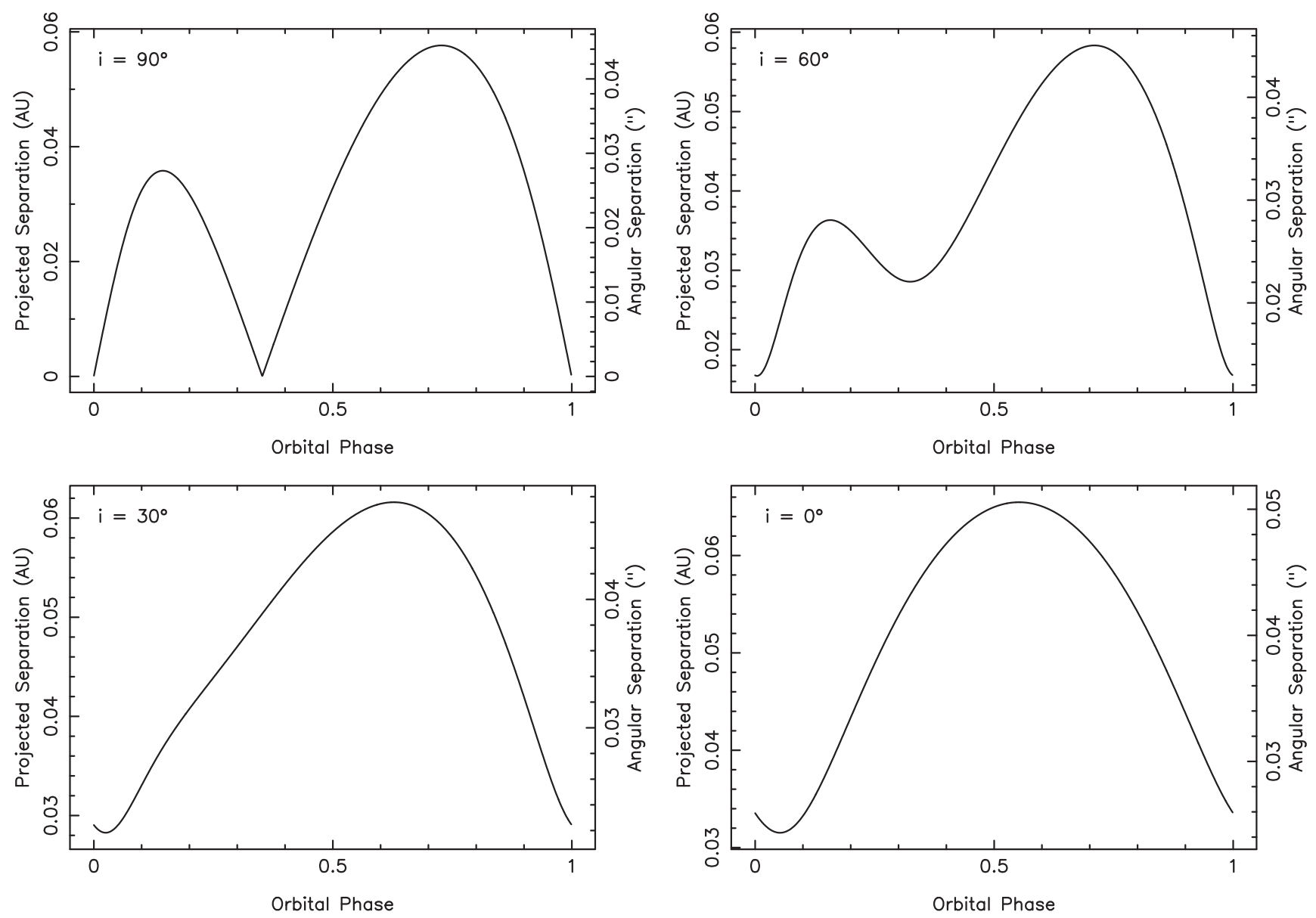

Figure 2. Projected and angular separation of Proxima Centauri b from the host star, assuming inclinations of $i=90^{\circ}$ (top left), $i=60^{\circ}$ (top right), $i=30^{\circ}$ (bottom left), and $i=0^{\circ}$ (bottom right). An orbital phase of zero corresponds to the location of superior conjunction. These separations need to be taken into account when planning future observations.

at quadrature points with SPHERE/ESPRESSO. Here we provide phase variation calculations as a function of orbital phase and inclination.

For reflected light at wavelength $\lambda$ and phase angle $\alpha$, the flux ratio of a planet with radius $R_{p}$ to the host star is given by

$$
\epsilon(\alpha, \lambda) \equiv \frac{f_{p}(\alpha, \lambda)}{f_{\star}(\lambda)}=A_{g}(\lambda) g(\alpha, \lambda) \frac{R_{p}^{2}}{r^{2}},
$$

where $A_{g}(\lambda)$ is the geometric albedo, $g(\alpha, \lambda)$ is the phase function, and $r$ is the star-planet separation. The value of $r$ depends upon the Keplerian orbital elements as follows:

$$
r=\frac{a\left(1-e^{2}\right)}{1+e \cos f}
$$

where $f$ is the true anomaly. The $R_{p}^{2} / r^{2}$ component of Equation (8) can thus become dominant for highly eccentric orbits. The phase angle, defined to be zero when the planet is at superior conjunction, is given by

$$
\cos \alpha=-\sin (\omega+f) .
$$

For the phase function $g(\alpha, \lambda)$, we adopt the empirically derived version of Hilton (1992), based upon observations of Jupiter and Venus. This approach uses a visual magnitude correction of the form

$$
\begin{aligned}
\Delta m(\alpha)= & 0.09\left(\alpha / 100^{\circ}\right) \\
& +2.39\left(\alpha / 100^{\circ}\right)^{2}-0.65\left(\alpha / 100^{\circ}\right)^{3}
\end{aligned}
$$

and the phase function is then given by

$$
g(\alpha)=10^{-0.4 \Delta m(\alpha)} .
$$

For the geometric albedo $A_{g}(\lambda)$, there are various values that could be adopted, such as the star-planet separation-dependent values of Kane \& Gelino (2010). For the purposes of this study, we adopt a value of 0.5 , which is midway between Earth $\left(A_{g}=0.367\right)$ and Venus $\left(A_{g}=0.67\right)$.

Shown in Figure 3 are the predicted changes in relative flux for Proxima Centauri b over one complete orbit, starting at a phase angle of $\alpha=0^{\circ}$. For completeness, we include the effects of Doppler boosting (Loeb \& Gaudi 2003; Faigler \& Mazeh 2011) and ellipsoidal variations (Morris \& Naftilan 1993; Zucker et al. 2007). The contributions to the total flux variations (solid line) shown in Figure 3 thus include the contributions from reflected light (dashed line), Doppler boosting (dotted-dashed line), and ellipsoidal variations (dotted line). As expected, the Doppler boosting and ellipsoidal variation components have negligible contributions since they depend largely upon the planetary mass. These calculations are performed for four different inclinations, ranging from edge-on $\left(i=90^{\circ}\right)$ to face-on $\left(i=1^{\circ}\right)$. 

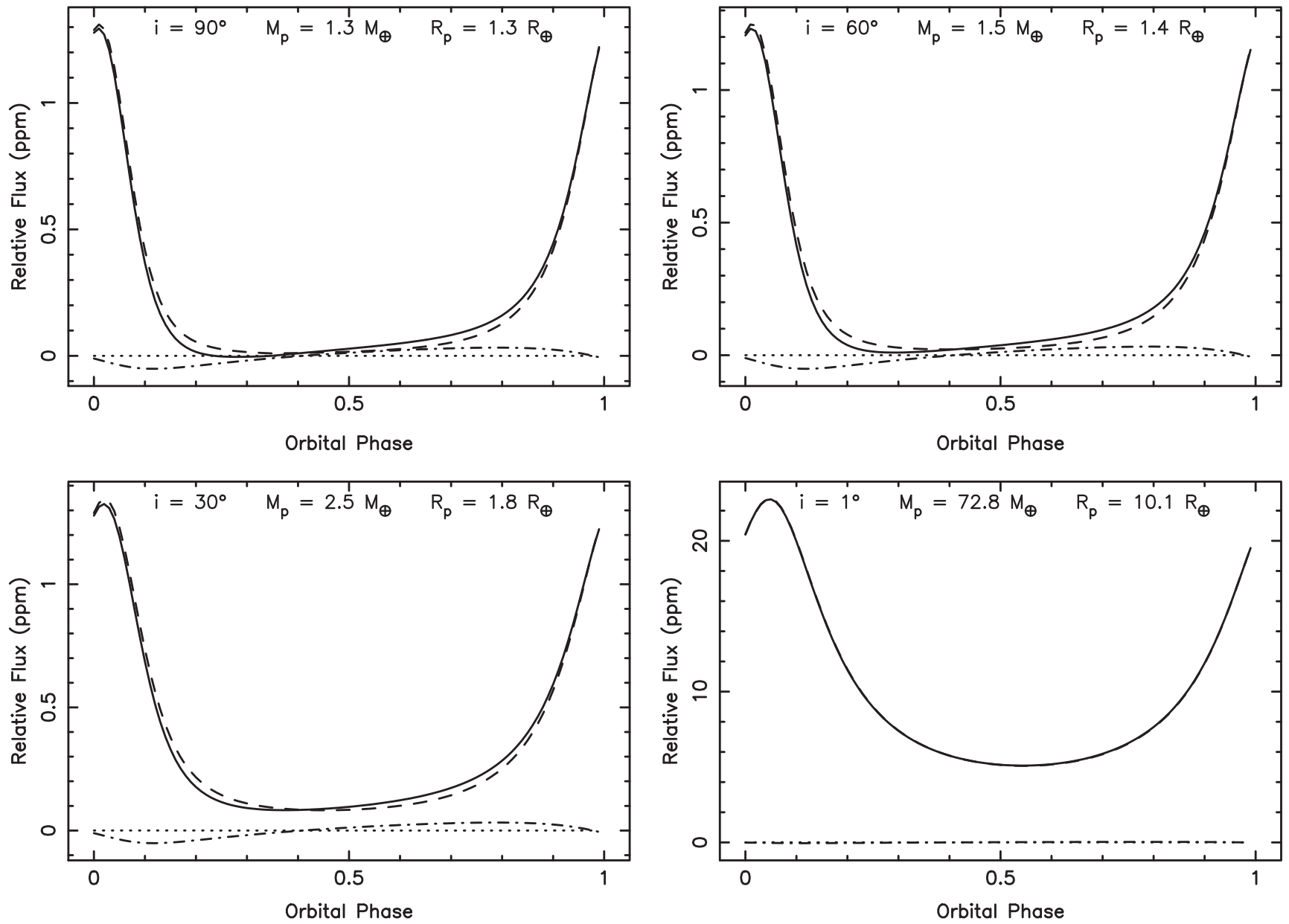

Figure 3. Flux ratio of Proxima Centauri $\mathrm{b}$ to the host star, assuming inclinations of $i=90^{\circ}$ (top left), $i=60^{\circ}$ (top right), $i=30^{\circ}$ (bottom left), and $i=1^{\circ}$ (bottom right). The flux is represented as parts per million (ppm). Shown are the contributions of reflected light (dashed line), Doppler boosting (dotted-dashed line), ellipsoidal variations (dotted line), and the combination of all three (solid line).
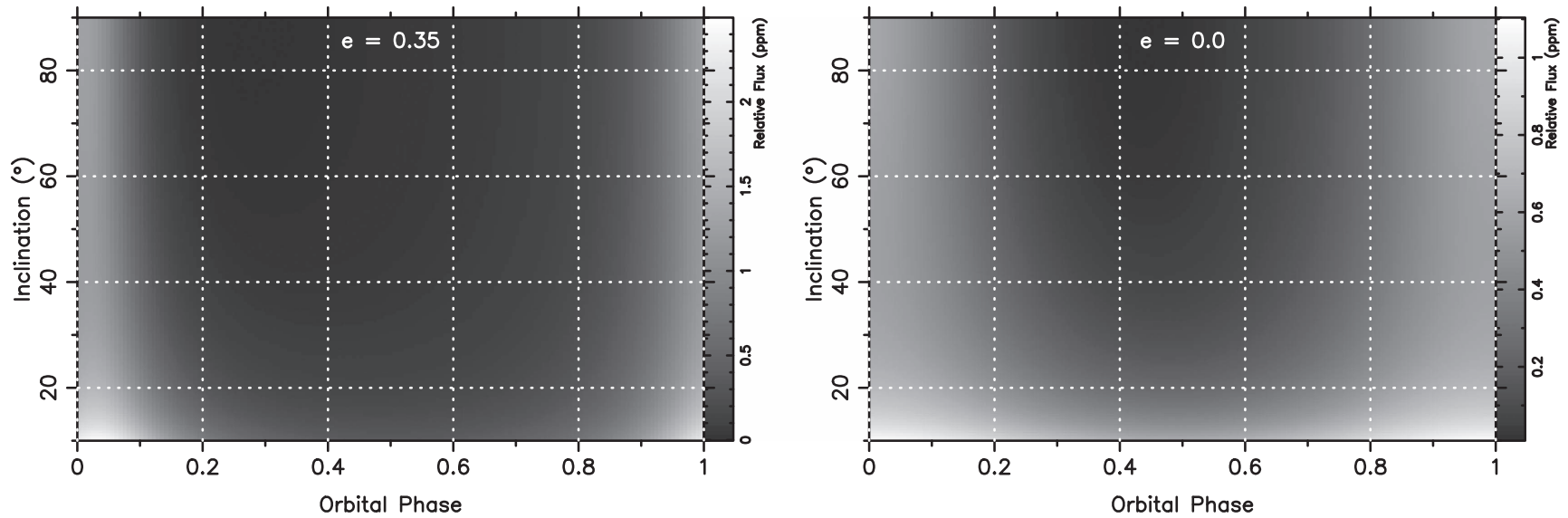

Figure 4. Intensity map for the flux ratio of Proxima Centauri $\mathrm{b}$ to the host star, as a function of orbital inclination and orbital phase for the eccentric case $(e=0.35)$ and the circular case $(e=0.0)$. The flux ratio includes the effects of reflected light, Doppler boosting, and ellipsoidal variations.

The primary change that occurs for the different inclinations is that the increase in planetary mass leads to an increase in radius, thus leading to an increase in flux ratio between the planet and star. To estimate the change in radius, we use the mass-radius relationship derived by Kane \& Gelino (2012b). Of particular interest is that, despite the loss of phase variations, the reflected light component dominates the total relative flux variations for face-on orbits due to the combination of large radius and orbital eccentricity. Figure 4 represents the orbital inclination dependence of the flux ratio profile as an intensity map, where the intensity scale is shown on the right of the figure. The peak flux ratio increases dramatically for orbital inclinations below $\sim 10^{\circ}$ due to the rise in planetary radius. The left and right panels of Figure 4 demonstrate the dramatic change in flux ratio as a function of orbital phase caused by the orbital eccentricity of the planet. 
Table 1

IR Contrast Ratios

\begin{tabular}{lcrrr}
\hline \hline $\begin{array}{l}\text { Inclination } \\
\left({ }^{\circ}\right)\end{array}$ & \multicolumn{4}{c}{ IR Contrast Ratio (ppm) } \\
\cline { 2 - 5 } & $3.6 \mu \mathrm{m}$ & $4.5 \mu \mathrm{m}$ & $8.0 \mu \mathrm{m}$ & $24.0 \mu \mathrm{m}$ \\
\hline 90 & 0.07 & 0.54 & 17.2 & 219.3 \\
60 & 0.08 & 0.63 & 20.0 & 254.3 \\
30 & 0.14 & 1.10 & 35.1 & 446.9 \\
1 & 4.31 & 34.44 & 1101.5 & 14006.8 \\
\hline
\end{tabular}

Thermal phase curves provide an additional avenue toward detection of the planet, depending on atmospheric composition and dynamics (Selsis et al. 2011; Maurin et al. 2012). For the planet-star contrast ratio at infrared wavelengths, we calculate their emissions assuming blackbody radiation and that the planetary atmosphere has $100 \%$ heat redistribution efficiency (Kane \& Gelino 2011b). The planetary equilibrium temperature is then given by

$$
T_{p}=\left(\frac{L_{\star}(1-A)}{16 \pi \sigma r^{2}}\right)^{\frac{1}{4}},
$$

where $L_{\star}$ is the stellar luminosity and $A$ is the planetary spherical (Bond) albedo. The observed contrast ratio at frequency $\nu$ is given by

$$
\frac{F_{p}}{F_{\star}}=\frac{\left(\exp \left(h \nu / k T_{\mathrm{eff}}\right)-1\right) R_{p}^{2}}{\left(\exp \left(h \nu / k T_{p}\right)-1\right) R_{\star}^{2}},
$$

where $T_{\text {eff }}$ is the stellar effective temperature. As for the phase variation calculations above, we assume a Bond albedo of $A=0.5$. We calculate contrast ratios for the original Spitzer passbands of $3.6,4.5,8.0$, and $24.0 \mu \mathrm{m}$ and for the four inclinations shown in Figure 3. These passbands are considered to be representative of the passbands that will be available at future facilities, such as the 2.4-5.0 $\mu \mathrm{m}$ wavelength range of NIRCam on the James Webb Space Telescope (JWST). The calculated contrast ratios are provided in Table 1 . It is clear from these numbers that the infrared flux of the planet will be readily detectable for inclinations less than $30^{\circ}$.

\section{HABITABLE ZONE AND ORBITAL STABILITY}

A further dependence of the mass of the known planet is the dynamical stability of additional terrestrial planets in or near the HZ of Proxima Centauri. To calculate the HZ, we use the stellar parameters of Anglada-Escudé et al. (2016) and the methodology of Kopparapu et al. (2013, 2014). This results in estimates for the "conservative" and "optimistic" HZ boundaries, the definitions of which depend upon assumptions regarding the prevalence of liquid water on the surfaces of Venus and Mars throughout their histories. For the conservative HZ, we calculate inner and outer boundaries of 0.041 and $0.081 \mathrm{au}$, respectively. For the optimistic HZ, we calculate inner and outer boundaries of 0.032 and $0.086 \mathrm{au}$, respectively. The extent of the HZ and the orbit of the known planet are depicted in the top-down view of the Proxima Centauri system shown in Figure 5. The conservative HZ is shown in light gray and the optimistic extension to the $\mathrm{HZ}$ is shown in dark gray. For the eccentric model of the orbit, the planet spends $93 \%$ of the orbital period within the $\mathrm{HZ}$, including the optimistic region.

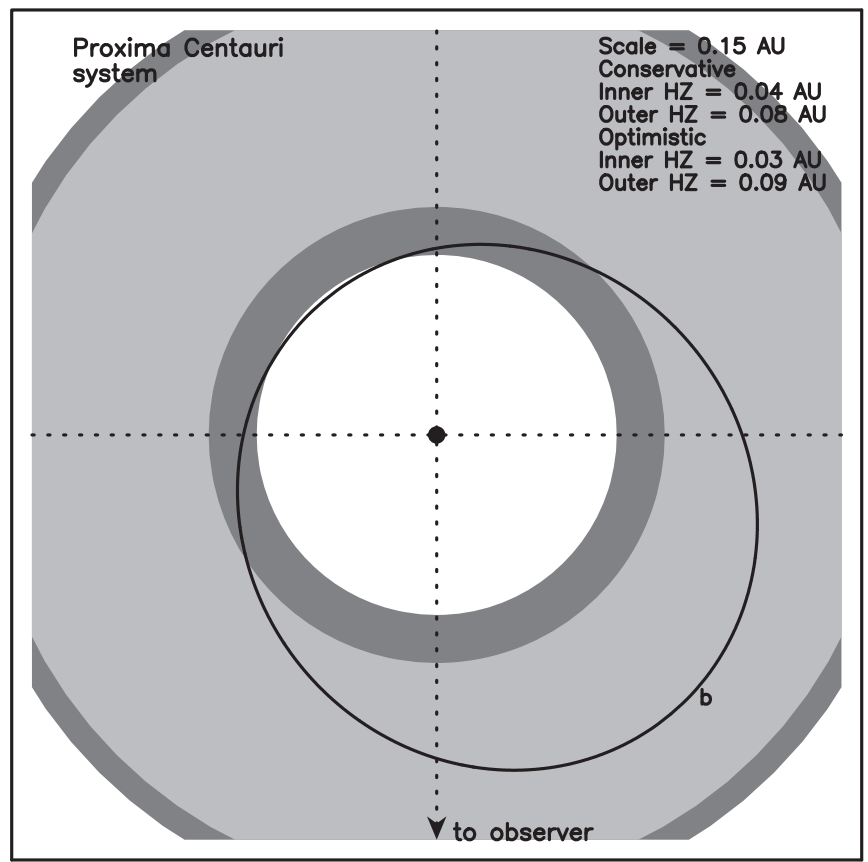

Figure 5. Top-down view of the Proxima Centauri system showing the extent of the $\mathrm{HZ}$ and orbits of the planets calculated using the stellar and planetary parameters from Anglada-Escudé et al. (2016). The physical scale depicted is $0.15 \mathrm{au}$ on a side. The conservative $\mathrm{HZ}$ is shown in light gray, and the optimistic extension to the $\mathrm{HZ}$ is shown in dark gray.

To test the orbital stability scenarios, we utilize the Mercury Integrator Package, described in detail by Chambers (1999). Mercury performs $N$-body integrations that are configured with user-supplied parameters that define the properties and starting conditions for the system. The specific integrator used was a hybrid symplectic/Bulirsch-Stoer integrator with a Jacobi coordinate system since that tends to provide greater accuracy for multiplanet systems (Wisdom \& Holman 1991; Wisdom 2006). We use a stability criterion that requires both planets to remain in the system for the duration of the simulation. If any of the planets are lost from the system, either by collision with the host star or ejection from the system, then the system is regarded as unstable.

We conducted a series of simulations that place an Earthmass planet as a test particle at a range of semimajor axes, from 0.02 to $0.1 \mathrm{au}$ in steps of $0.005 \mathrm{au}$. Such an orbital range fully encompasses both the orbit of the known planet and the HZ of the system. We assumed a circular orbit for the additional planet and used a time resolution of 0.1 days to ensure that the minimum time-step recommendation of Duncan et al. (1998; $1 / 20$ of the shortest system orbital period) was met at all times. The known planet was assumed to have the maximum allowed eccentricity of $e=0.35$. The simulations were conducted for three different inclination scenarios of $90^{\circ}, 30^{\circ}$, and $10^{\circ}$. These inclinations imply a mass for the known planet of 1.27, 2.54, and $7.31 M_{\oplus}$, respectively.

The outcome of the orbital stability simulations are shown in Figure 6, where the separate panels show the results for the $i=90^{\circ} \quad$ (top), $i=30^{\circ} \quad$ (middle), and $i=10^{\circ}$ (bottom) scenarios. As for Figure 5, the conservative $\mathrm{HZ}$ is shown in light gray and the optimistic extension to the $\mathrm{HZ}$ is shown in dark gray. For $i=90^{\circ}$, the presence of the known planet excludes other planets within the $\mathrm{HZ}$ with the exception of the locations of mean-motion resonance (MMR), shown in Figure 6 

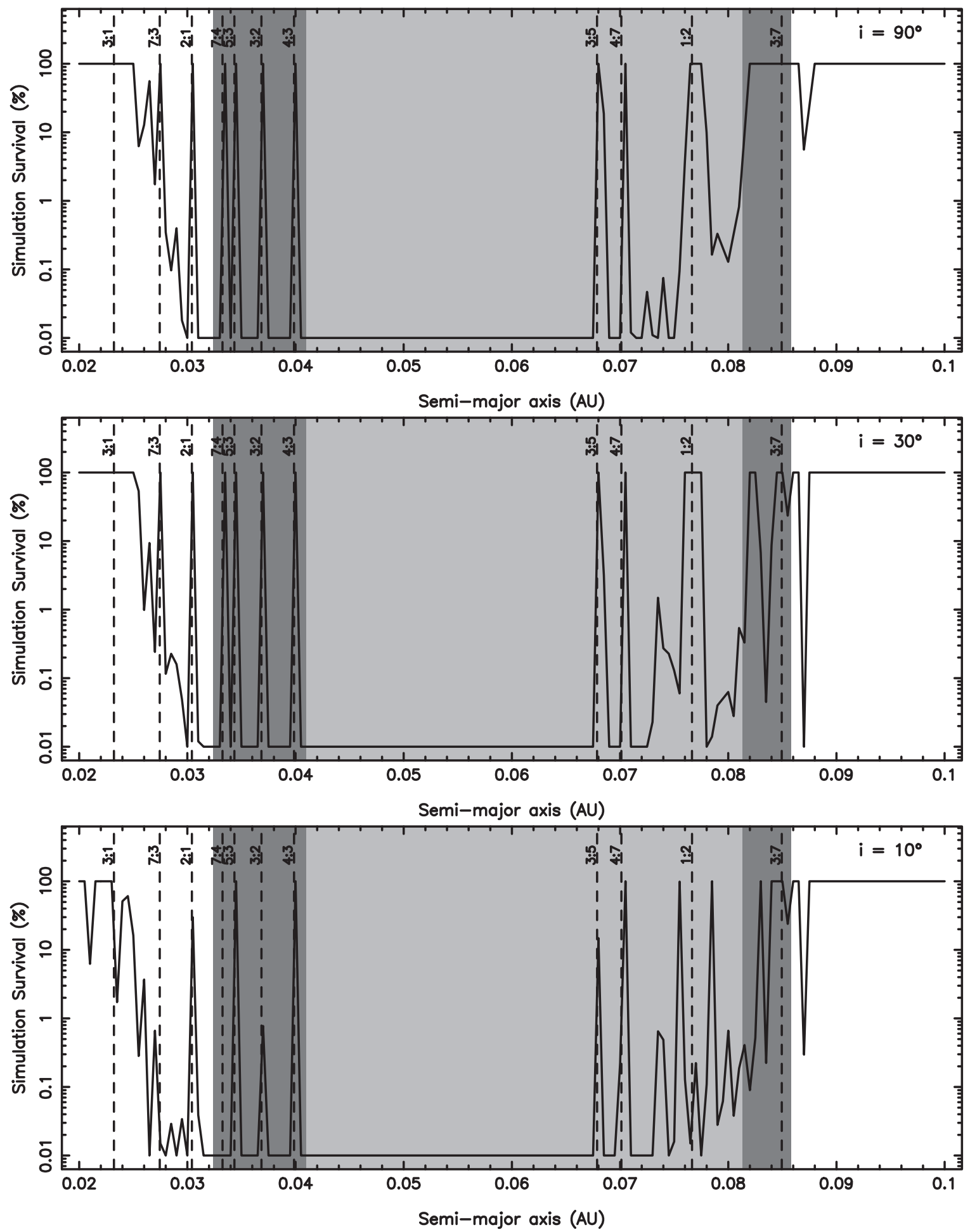

Figure 6. Orbital stability of a hypothetical Earth-mass planet as a function of semimajor axis in the Proxima Centauri system. The individual panels show the results for simulations that assume the known planet has an inclination of $90^{\circ}$ (top), $30^{\circ}$ (middle), and $10^{\circ}$ (bottom). These inclinations affect the true mass of the known planet and thus the overall stability of the system. The orbital stability on the vertical axis is expressed as the percentage simulation survival for each semimajor axis where the position of the planet was tested. The system is assumed to be coplanar and the orbit of the Earth-mass planet is assumed to be circular. The light-gray and dark-gray regions represent the conservative and optimistic HZ regions, as per Figure 5. The vertical dashed lines indicate the locations of the mean-motion orbital resonances with the known planet.

as vertical dashed lines. Outside of the HZ, the dynamical viability of additional planets rises dramatically. There is very little difference between the $90^{\circ}$ and $30^{\circ}$ cases, since the Hill radius has a mass dependence of $M_{p}^{(1 / 3)}$ but scales linearly with $a$. Thus, the close proximity of the planet(s) to the host star dominates the orbital dynamics and subsequent stability, as observed for compact systems, such as those found by Kepler (Raymond et al. 2009). Note however that the 3:7 MMR narrows significantly between the $90^{\circ}$ and $30^{\circ}$ cases, reducing its viability as an orbital location for another planet. 
The third scenario that we investigated was the case for $i=10^{\circ}$, where the mass of the known planet would be $\sim 7.31 M_{\oplus}$. The result of this simulation is shown in the bottom panel of Figure 6 . The main effect of the planetary mass increase is to further erode the significance of the MMR locations, rendering them largely unstable. The exception to this is the emergence of two stability locations on either side of the 1:2 MMR. An additional effect of the interaction between the two planets is the exchange of angular momentum, resulting in oscillating eccentricities of the known planet (for example, see Kane \& Raymond 2014). Tidal effects will also undoubtedly play a role in the potential habitability of the known planet (Barnes et al. 2009) as well as circularizing the orbit (Barnes et al. 2016). If the eccentricity of the known planet is close to the maximum of 0.35 found by AngladaEscudé et al. (2016), the planet may spend extended periods in a close-to-circular orbit within the $\mathrm{HZ}$ as the other planet increases in eccentricity.

Finally, we tested the case of a circular $(e=0.0)$ edge-on $\left(i=90^{\circ}\right)$ orbit for Proxima Centauri $\mathrm{b}$ with the addition of the hypothetical Earth-mass planet described above. In this case, the orbital stability of the system is preserved for all semimajor axes of the additional planet outside of the range 0.044-0.053 au. Comparison of this range with the instability regions depicted in Figure 6 shows that a circular orbit for planet $\mathrm{b}$ allows there to be significantly more locations where another low-mass planet could be harbored by the system in a stable orbit than for the eccentric case. This result emphasizes the dependence on orbital eccentricity and the need to fully understand the Keplerian nature of the planet $b$ orbit.

\section{OBSERVATIONAL PROSPECTS}

\subsection{Near-term Characterization}

Clearly, further observations are required to resolve the inclination ambiguity described in this work and determine whether this world could be habitable. In Section 3, we demonstrated that an astrometric signal for this planet is within the sensitivity regime of Gaia, if the planet is a gas giant. Thus we may have confirmation of the planet's terrestrial nature within the next several years of Gaia data releases. Meanwhile, JWST may offer a near-term prospect for constraining the planet's size and atmospheric thermal properties for lowinclination orbits (see Section 5). Kriedberg \& Loeb (2016) further find that with complete phase coverage, JWST could detect variations in thermal emission with a precision sufficient to distinguish between bare rock and a planet with partially $(35 \%)$ redistributed heat due to the presence of an atmosphere and/or ocean.

\subsection{Direct Imaging from the Ground}

Ultimately, the greatest promise for assessing the habitability (or inhabitance) of Proxima Centauri b lies with directly imaging the planet and determining atmospheric composition via spectral characterization. In Section 3, we calculated a maximum angular separation between Proxima Centauri and planet $\mathrm{b}$ of $\sim 65$ mas, depending on inclination and eccentricity. In Section 5, we calculate a planet-to-star flux ratio in reflected starlight of $10^{-5}-10^{-6}$, with flux variations at levels of $10^{-6}$ or more over the course of an orbit, due to phase changes.

To date, this star-planet separation and flux contrast is substantially smaller than what has been achieved with direct imaging even for young, self-luminous planets (e.g., Macintosh et al. 2015). As mentioned in Section 5, Lovis et al. (2016) have suggested the possibility of directly imaging Proxima Centauri $b$ by combining the SPHERE coronagraph with the ESPRESSO spectrograph at the $8 \mathrm{~m}$ ESO VLT. They calculate a detection time of 20-40 nights of telescope time and possible atmospheric $\mathrm{O}_{2}$ detection in 60 nights. In the more distant future, extremely large ( $\sim 40 \mathrm{~m}$ class) ground-based observatories could offer the capability required to spectrally characterize this planet in the optical and near-IR. However, Meadows et al. (2016) point out that shorter wavelength coverage than is currently planned for the E-ELT and GMT adaptive optics capabilities would be desirable.

\subsection{Direct Imaging from Space}

Space-based coronograph and/or starshade missions may provide the greatest capability in revealing the nature of the Proxima Centauri planet through spectral characterization in the UV through near-IR. The Wide-field Infrared Survey Telescope (WFIRST) will be the first technology demonstration of wavefront-controlled space-based exoplanet imaging coronagraphs, and it is scheduled for launch in $\sim 2025$. The highest priority WFIRST targets will be chosen from the brightest known RV planets. The most ambitious of the WFIRST coronagraph designs was the phase-induced amplitude apodization complex mask coronagraph (PIAACMC). PIAACMC's assumed inner working angle and contrast floor (40 mas and $\sim 3.4 \times 10^{-10}$; Traub et al. 2016) would have been sufficient to detect the planet at locations near the maximum angular separation found in this paper. However, PIAACMC was designated as a "backup" instrument due to outstanding technical challenges, and it is unlikely to advance beyond Phase A. The baseline WFIRST Hybrid Lyot and Shaped Pupil coronagraphs will achieve inner working angles of 120-150 mas in the shortest wavelength band at $465 \mathrm{~nm}$, but this performance is not sufficient to image Proxima Centauri b.

The WFIRST baseline mission also includes starshade readiness, which leaves open the possibility that a separately launched starshade could rendezvous with WFIRST later in the mission. Seager et al. (2015) found that such a rendezvous mission could achieve an inner working angle of $\sim 70$ mas in the bluest bandpass (425-600 nm), and a contrast limit of better than $10^{-10}$. This is approaching the necessary inner working angle required for detecting Proxima Centauri b, and perhaps the design could be further optimized for this target. However, the large size of the WFIRST point-spread function ( $~ 50$ mas) may nevertheless lead to prohibitively long exposure times even with the high throughput provided by a starshade.

Therefore, we conclude that the spectral characterization of Proxima Centauri b in the UV through near-IR may have to wait for larger space-based concepts like the 4-6.5 m "Hab-Ex" Habitable Exoplanets Imaging Mission ${ }^{6}$ or the $8-12 \mathrm{~m}$ LUVOIR $^{7}$ currently under study. It is worth noting that a prerequisite for scheduling observations times is the refinement of the planetary orbit to produce an accurate ephemeris (Kane et al. 2009).

\footnotetext{
6 http://www.jpl.nasa.gov/habex/

7 http://cor.gsfc.nasa.gov/studies/luvoir.php
} 


\section{CONCLUSIONS}

Proxima Centauri $b$ is the closest exoplanet to our planetary system, and thus provides interesting prospects for further characterization. The terrestrial nature of the planet is quite likely, and is calculated by us to be $\sim 84 \%$ (see Section 2). Furthermore, previous studies have shown that giant planets in short-period orbits around $\mathrm{M}$ dwarfs are relatively rare (Bonfils et al. 2013; Tuomi et al. 2014; Dressing \& Charbonneau 2015). However, the ambiguity regarding the mass of the planet due to the unknown orbital inclination will greatly influence the outcome of further investigations. The mass of the planet will have a profound impact on such other properties as radius, atmospheric scale height, composition, and albedo. Each of these properties, in turn, will determine the detectability of the planet via alternative methods.

In this work, we have quantified the astrometric signature and angular separation of the planet as a function of inclination. Our astrometry calculations show the region of inclinations and astrometric amplitudes where the planet can be considered to have crossed from the terrestrial into the gas giant regime. Although a close to face-on inclination would entail the planet not being of terrestrial mass, the angular separation calculations show that this scenario produces the largest angular separation and the least constraints on direct imaging observations.

Our calculations of the expected phase variations as a function of inclination show that the face-on scenarios produce the largest amplitude. However, face-on phase amplitudes are being driven by a large reflecting area (radius) and a timedependent star-planet separation, and so depends highly upon the eccentricity of the orbit. For inclinations where $i>30^{\circ}$, there is very little difference in the overall shape and amplitude of the phase variations, including the components of reflected light, Doppler boosting, and ellipsoidal variations. The change in inclination has a dramatic effect on the predicted contrast ratio at infrared wavelengths, such as a contrast ratio of $\sim 1 \%$ at $24 \mu \mathrm{m}$ for an inclination of $i=1^{\circ}$.

We calculated the extent of the optimistic and conservative $\mathrm{HZ}$ for Proxima Centauri and conducted exhaustive dynamical simulations to determine the viability of other terrestrial planets within the $\mathrm{HZ}$ region. Our simulations demonstrate that the presence of the known planet with an eccentric orbit excludes the possibility of another terrestrial planet throughout most of the HZ, with the exception of MMR locations. Reducing the inclination to $i=10^{\circ}$ further compounds the instability within the $\mathrm{HZ}$ regions.

The overall results contained within this work are meant to serve as a guide for future observations intended to characterize the planet, particularly those that may detect reflected light or direct emission from the planet. Such observations may include proposed coronographs or similar instruments for future spacebased imaging missions. Other techniques beyond those discussed here, such as microlensing (Sahu et al. 2014), may also benefit from our quantification of the observable signatures. Given that the Proxima Centauri planet is not only the closest exoplanet but the nearest planet in the $\mathrm{HZ}$ of its host star, the potential rewards highly warrant further studies.

The authors would like to thank the anonymous referee, whose comments improved the quality of the paper. Thanks are also due to Guillem Anglada-Escudé and Franck Selsis for their useful feedback on the manuscript. This research has made use of the following archives: the Exoplanet Orbit Database and the
Exoplanet Data Explorer at exoplanets.org, the Habitable Zone Gallery at hzgallery.org, and the NASA Exoplanet Archive, which is operated by the California Institute of Technology, under contract with the National Aeronautics and Space Administration under the Exoplanet Exploration Program. The results reported herein benefited from collaborations and/or information exchange within NASA's Nexus for Exoplanet System Science (NExSS) research coordination network sponsored by NASA's Science Mission Directorate.

\section{REFERENCES}

Anglada-Escudé, G., Amado, P. J., Barnes, J., et al. 2016, Natur, 536, 437

Barnes, R., Deitrick, R., Luger, R., et al. 2016, AsBio, submitted (arXiv:1608. 06919)

Barnes, R., Jackson, B., Greenberg, R., \& Raymond, S. N. 2009, ApJL, 700, L30

Bonfils, X., Delfosse, X., Udry, S., et al. 2013, A\&A, 549, A109

Brown, A. G. A., Vallenari, A., Prusti, T., et al. 2016, A\&A, 595, A2

Chambers, J. E. 1999, MNRAS, 304, 793

Coleman, G. A. L., Nelson, R. P., Paardekooper, S.-J., et al. 2016, MNRAS, submitted (arXiv:1608.06908)

Collins, J. M., Jones, H. R. A., \& Barnes, J. R. 2016, A\&A, submitted (arXiv:1608.07834)

Davenport, J. R. A., Kipping, D. M., Sasselov, D., Matthews, J. M., \& Cameron, C. 2016, ApJL, 829, L31

de Bruijne, J. H. J. 2012, Ap\&SS, 341, 31

Dressing, C. D., \& Charbonneau, D. 2015, ApJ, 807, 45

Dressing, C. D., Charbonneau, D., Dumusque, X., et al. 2015, ApJ, 800, 135

Duncan, M. J., Levison, H. F., \& Lee, M. H. 1998, AJ, 116, 2067

Faigler, S., \& Mazeh, T. 2011, MNRAS, 415, 3921

Hilton, J. L. 1992, in Explanatory Supplement to the Astronomical Almanac, ed. P. K. Seidelmann (Mill Valley, CA: Univ. Science Books), 383

Kane, S. R. 2013, ApJ, 766, 10

Kane, S. R., \& Gelino, D. M. 2010, ApJ, 724, 818

Kane, S. R., \& Gelino, D. M. 2011a, ApJ, 729, 74

Kane, S. R., \& Gelino, D. M. 2011b, ApJ, 741, 52

Kane, S. R., \& Gelino, D. M. 2012a, MNRAS, 424, 779

Kane, S. R., \& Gelino, D. M. 2012b, PASP, 124, 323

Kane, S. R., Hill, M. L., Kasting, J. F., et al. 2016, ApJ, 830, 1

Kane, S. R., Mahadevan, S., von Braun, K., Laughlin, G., \& Ciardi, D. R. 2009, PASP, 121, 1386

Kane, S. R., \& Raymond, S. N. 2014, ApJ, 784, 104

Kipping, D. M., Cameron, C., Hartman, J. D., et al. 2016, ApJ, in press (arXiv:1609.08718)

Kopparapu, R. K., Ramirez, R., Kasting, J. F., et al. 2013, ApJ, 765, 131

Kopparapu, R. K., Ramirez, R. M., SchottelKotte, J., et al. 2014, ApJL, 787, L29

Kriedberg, L., \& Loeb, A. 2016, ApJL, 832, L12

Lindegren, L., Lammers, U., Bastian, U., et al. 2016, A\&A, 595, A4

Loeb, A., \& Gaudi, B. S. 2003, ApJL, 588, L117

Lovis, C., Snellen, I., Mouillet, D., et al. 2016, A\&A, submitted (arXiv:1609. 03082)

Macintosh, B., Graham, J. R., Barman, T., et al. 2015, Sci, 350, 64

Madhusudhan, N., Agúndez, M., Moses, J. I., \& Hu, Y. 2016, SSRv, in press (arXiv:1604.06092)

Maurin, A. S., Selsis, F., Hersant, F., \& Belu, A. 2012, A\&A, 538, A95

Mayor, M., Udry, S., Lovis, C., et al. 2009, A\&A, 493, 639

Meadows, V. S., Arney, G. N., Schwieterman, E. W., et al. 2016, AsBio, submitted (arXiv:1608.08620)

Morris, S. L., \& Naftilan, S. A. 1993, ApJ, 419, 344

O’Malley-James, J. T., \& Kaltenegger, L. 2016, ApJ, submitted (arXiv:1608. 06930)

Perryman, M., Hartman, J., Bakos, G. A., \& Lindegren, L. 2014, ApJ, 797, 14 Prusti, T., de Bruijne, J. H. J., Brown, A. G. A., et al. 2016, A\&A, 595, A1 Raymond, S. N., Barnes, R., Veras, D., et al. 2009, ApJL, 696, L98

Ribas, I., Bolmont, E., Selsis, F., et al. 2016, A\&A, 596, A111

Robertson, P., Bender, C., Mahadevan, S., Roy, A., \& Ramsey, L. W. 2016, ApJ, 832, 112

Rogers, L. A. 2015, ApJ, 801, 41

Sahu, K. C., Bond, H. E., Anderson, J., \& Dominik, M. 2014, ApJ, 782, 89

Seager, S., Turnbull, M., Sparks, W., et al. 2015, Proc. SPIE, 9605, 96050W

Seager, S., Whitney, B. A., \& Sasselov, D. D. 2000, ApJ, 540, 504

Selsis, F., Wordsworth, R. D., \& Forget, F. 2011, A\&A, 532, A1 
Sudarsky, D., Burrows, A., Hubeny, I., \& Li, A. 2005, ApJ, 627, 520

Traub, W. A., Breckinridge, J., Greene, T. P., et al. 2016, JATIS, 2, 11020

Tuomi, M., Anglada-Escudé, G., Gerlach, E., et al. 2013, A\&A, 549, A48

Tuomi, M., Jones, H. R. A., Barnes, J. R., Anglada-Escudé, G., \& Jenkins, J. S. 2014, MNRAS, 441, 1545

Tuomi, M., Kotiranta, S., \& Kaasalainen, M. 2009, A\&A, 494, 769
Turbet, M., Leconte, J., Selsis, F., et al. 2016, A\&A, 596, A112

Weiss, L. M., \& Marcy, G. W. 2014, ApJL, 783, L6

Wisdom, J. 2006, AJ, 131, 2294

Wisdom, J., \& Holman, M. 1991, AJ, 102, 1528

Zakamska, N. L., Pan, M., \& Ford, E. B. 2011, MNRAS, 410, 1895

Zucker, S., Mazeh, T., \& Alexander, T. 2007, ApJ, 670, 1326 